\title{
CHROMATICITY FOR ENERGY SPREAD MEASUREMENT AND FOR CURE OF TRANSVERSE MULTI-BUNCH INSTABILITY IN THE SPRING-8 STORAGE RING
}

\author{
T. Nakamura, K. Soutome, M. Takao, S. Takano T. Ohshima, M. Masaki, S. Sasaki, \\ M. Shoji, K. Tsumaki, JASRI/SPring-8, Mikazuki-cho, Hyogo, JAPAN
}

\section{Abstract}

Chromaticity of a ring introduces non-harmonic betatron motion to off-momentum electrons. This motion produces synchrotron sidebands of betatron frequency in frequency response of betatron motion of a beam and reduces the main peak height. The height of these peaks depends on chromaticity and on the energy spread of the beam and we measured energy spread of the SPring- 8 storage ring.

The reduction of the main peak works to reduce the strength of instabilities and we suppress the horizontal coupled-bunch instability driven by cavity higher order modes and the vertical coupled-bunch instability driven by resistive-wall impedance of the in-vacuum type insertion devices by introducing horizontal and vertical chromaticity 7 and 6 , respectively. These chromaticity reduce the strength of these instabilities to half of those at zero chromaticity.

\section{INTRODUCTION}

Finite chromaticity of storage rings produces the synchrotron sideband peaks in frequency response of betatron motion and reduces the main peak height. These peak heights depend on chromaticity and on energy spread. The value of chromaticity can be easily estimated from the dependence of betatron tune on the energy shift controlled by RF acceleration frequency and we can obtain the energy spread of the beam. The reduction of the height of main peak by chromaticity increases the resistivity of the beam to coupled-bunch instabilities and we suppress the horizontal coupled-bunch instability driven by higher order modes of RF cavities and the vertical coupled-bunch instability driven by resistive-wall impedance of the in-vacuum type insertion devices. For both instabilities, the threshold current at zero chromaticity is less than half of the operation current which we obtain by setting chromaticity to nearly 7 .

\section{PEAK HEIGHT OF SYNCHROTRON SIDEBAND}

The example of measured frequency response of a betatron motion of a stored beam in the SPring- 8 storage ring is shown in Fig.1 and we can see several synchrotron sideband peaks around a main peak of betatron frequency. First, we show the expression of the relative peak height of synchrotron sideband to the main peak height.

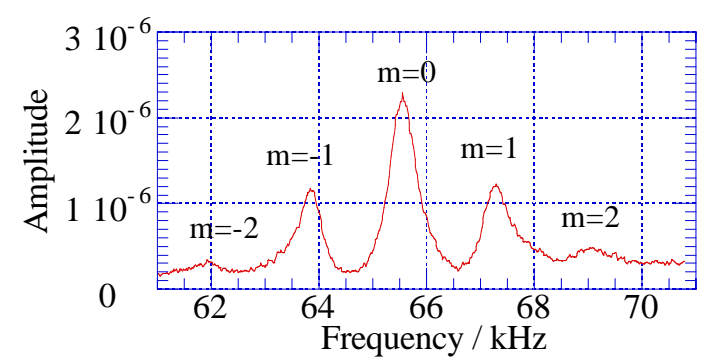

Figure 1: Example of measured frequency response of vertical betatron motion of a beam in the SPring- 8 storage ring. Chromaticity is 8 and synchrotron frequency is $1.63 \mathrm{kHz}$.

The equation of betatron motion of an electron with energy oscillation $\delta=r \cos \left(\omega_{\mathrm{s}} \mathrm{t}+\phi\right)$ in finite chromaticity $\xi$ is

$$
\begin{aligned}
& \frac{d^{2} q}{d t^{2}}+2 \alpha \frac{d q}{d t}+\omega(t)^{2} q=F(t) \\
& \omega(t)=\omega_{\beta}+\omega_{0} \xi r \cos \left(\omega_{s} t+\phi\right) \\
& F(t)=c \hat{\theta} \cos \left(\omega_{f} t+\psi\right) \sum_{k=-\infty}^{\infty} \delta\left(t-k T_{0}\right) \\
& =c \hat{\theta} \cos \left(\omega_{f} t+\psi\right) \frac{1}{T_{0}}\left(1+2 \sum_{p=1}^{\infty} \cos p \omega_{0} t\right)
\end{aligned}
$$

where $\mathrm{F}$ is driven by a shaker of kick angle amplitude $\hat{\theta}$ and $\omega_{0}$ is angular revolution frequency. We assume that $\omega_{\mathrm{f}} \sim \omega_{\beta}$ and we take the base band of F. You may choose $\omega_{\mathrm{f}} \sim \omega_{\beta}-\mathrm{p} \omega_{0}$ and take a term $\cos \left(\omega_{\mathrm{f}} \mathrm{t}+\psi\right) \cos \left(\mathrm{p} \omega_{0} \mathrm{t}\right)$ instead. The origin of time $\mathrm{t}$ is chosen to $\psi=0$ in following discussion.

The Green function of Eq.(1) is

$$
\begin{aligned}
G_{0}\left(t, t^{\prime}\right) & =-\frac{i}{\sqrt{\omega(t) \omega\left(t^{\prime}\right)}} e^{-\alpha\left(t-t^{\prime}\right)+i \int_{t^{\prime}}^{t} \omega^{\prime}\left(t^{\prime \prime}\right) d t^{\prime \prime}} \\
& \cong-\frac{i}{\omega_{\beta}} e^{-\alpha t+i \int_{0}^{t} \omega^{\prime}\left(t^{\prime \prime}\right) d t^{\prime \prime}} e^{\alpha t-i \int_{0}^{t^{\prime}} \omega^{\prime}\left(t^{\prime \prime}\right) d t^{\prime \prime}}
\end{aligned}
$$

The Green function which include the timing modulation produced by the synchrotron motion is

$$
\begin{aligned}
& G\left(t, t^{\prime}, r, \phi\right)=G_{0}\left(t-\tau(t), t^{\prime}-\tau\left(t^{\prime}\right)\right) \\
& =G_{0}\left(t+\frac{\alpha}{\omega_{s}} r \sin \left(\omega_{s} t+\phi\right), t^{\prime}+\frac{\alpha}{\omega_{s}} r \sin \left(\omega_{s} t^{\prime}+\phi\right)\right)
\end{aligned}
$$




$$
\begin{aligned}
& \cong-\frac{i}{\omega_{\beta}} e^{-\alpha t+i \int_{0}^{t+\frac{\alpha}{\omega_{s}} r \sin \left(\omega_{s} t+\phi\right)} \omega^{\prime}\left(t^{\prime \prime}\right) d t^{\prime \prime}} \\
& \times e^{\alpha t-i \int_{0}^{t^{\prime}+\frac{\alpha}{\omega_{s}} r \sin \left(\omega_{s} t^{\prime}+\phi\right)} \omega^{\prime}\left(t^{\prime \prime}\right) d t^{\prime \prime}}
\end{aligned}
$$

. Using the expansion to a series of Bessel functions;

$$
\begin{gathered}
e^{i \int_{0}^{t+\frac{\alpha}{\omega_{s}} r \sin \left(\omega_{s} t+\phi\right)} \omega^{\prime}\left(t^{\prime \prime}\right) d t^{\prime \prime}}=e^{i \omega_{\beta} t} e^{i\left(\frac{\alpha}{\omega_{s}} \omega_{\beta}+\frac{\omega_{0} \xi}{\omega_{s}}\right) r \sin \left(\omega_{s} t+\phi\right)} \\
=e^{i \omega_{\beta} t} \sum_{n=-\infty}^{\infty} J_{n}\left(\left(\frac{\alpha}{\omega_{s}} \omega_{\beta}+\frac{\omega_{0} \xi}{\omega_{s}}\right) r \xi^{i n \omega_{s} t} e^{i \phi}\right.
\end{gathered}
$$

, we have

$$
\begin{gathered}
G\left(t, t^{\prime}, r, \phi\right)=-\frac{i}{\omega_{\beta}} e^{\left(-\alpha+i \omega_{\beta}\right)\left(t-t^{\prime}\right)} \\
\times \sum_{m=-\infty}^{\infty} \sum_{m^{\prime}=-\infty}^{\infty} J_{m}\left(\left(\frac{\omega_{\beta} \alpha}{\omega_{s}}+\frac{\omega_{0} \xi}{\omega_{s}}\right)\right) J_{m^{\prime}}\left(\left(\frac{\omega_{\beta} \alpha}{\omega_{s}}+\frac{\omega_{0} \xi}{\omega_{s}}\right)\right) \\
\times e^{i m \omega_{s} t} e^{-i m^{\prime} \omega_{s} t^{\prime}} e^{i\left(m-m^{\prime}\right) \phi}
\end{gathered}
$$

- Green function for a bunch with Gaussian energy distribution is

$$
\begin{gathered}
\left\langle G\left(t, t^{\prime}, r, \phi\right)\right\rangle=\frac{1}{2 \pi \sigma_{\delta}^{2}} \int_{0}^{2 \pi} d \phi \int_{0}^{\infty} r d r G\left(t, t^{\prime}, r, \phi\right) e^{-\frac{r^{2}}{2 \sigma_{\delta}^{2}}} \\
=-\frac{i}{\omega_{\beta}} \frac{1}{\sigma_{\delta}^{2}} \sum_{m=-\infty}^{\infty} \int_{0}^{\infty} J_{m}^{2}\left(\left(\frac{\omega_{\beta} \alpha}{\omega_{s}}+\frac{\omega_{0} \xi}{\omega_{s}}\right)\right) e^{-\frac{r^{2}}{2 \sigma_{\delta}^{2}}} r d r \\
\times e^{\left(-\alpha+i\left(\omega_{\beta}+m \omega_{s} t\right)\right)\left(t-t^{\prime}\right)}
\end{gathered}
$$

where $\sigma_{\delta}$ is the r.m.s. relative energy spread and we assume $\omega(t) \sim \omega_{\beta}$. Using this, we have the motion of the bunch,

$$
\begin{aligned}
\langle q(t)\rangle & =\int_{-\infty}^{t}\left\langle G\left(t, t^{\prime}, r, \phi\right)\right\rangle F\left(t^{\prime}\right) d t^{\prime} \\
& =-\frac{c \hat{\theta}}{2 T_{0}} \frac{i}{\omega_{\beta}} \frac{1}{\sigma_{\delta}^{2}} \sum_{m=-\infty}^{\infty} \int_{0}^{\infty} J_{m}^{2}\left(\left(\frac{\omega_{\beta} \alpha}{\omega_{s}}+\frac{\omega_{0} \xi}{\omega_{s}}\right)\right) e^{-\frac{r^{2}}{2 \sigma_{\delta}^{2}}} r d r \\
& \times\left(\frac{e^{i \omega_{f} t}}{\alpha-i\left(\omega_{\beta}+m \omega_{s}-\omega_{f}\right)}+\frac{e^{-i \omega_{f} t}}{\alpha-i\left(\omega_{\beta}+m \omega_{s}+\omega_{f}\right)}\right)
\end{aligned}
$$

(8)

With this, the peak height at resonance $\omega_{\mathrm{f}}=\omega_{\beta}+\mathrm{n} \omega_{\mathrm{s}}$ is

$$
\left\langle\tilde{q}\left(\omega_{\beta}+m \omega_{s}\right)\right\rangle=-i \frac{\sqrt{\beta \beta_{0}} \hat{\theta}}{2 T_{0} \alpha} \frac{1}{\sigma_{\delta}^{2}} \int_{0}^{\infty} J_{m}^{2}\left(\left(\frac{\omega_{\beta} \alpha}{\omega_{s}}+\frac{\omega_{0} \xi}{\omega_{s}}\right)\right) e^{-\frac{r^{2}}{2 \sigma_{\delta}^{2}}} r d r
$$

where we assumed the distance between peaks is far enough not to interfere each other. In Eq.(6), we replaced $c / \omega_{\beta}$ with the beta functions; $\beta_{0}$ and $\beta$, at the shaker and a observation point, respectively. The relative peak height to the height of the $\mathrm{m}=0$ peak at $\xi=0$ is

$$
R_{m}(y)=\frac{1}{y^{2}} \int_{0}^{\infty} J_{m}^{2}(x) e^{-\frac{x^{2}}{2 y^{2}}} x d x
$$

where $y=\left(\frac{\omega_{\beta} \alpha}{\omega_{s}}+\frac{\omega_{0} \xi}{\omega_{s}}\right) \sigma_{\delta}$. This is shown in Fig. 2-3.

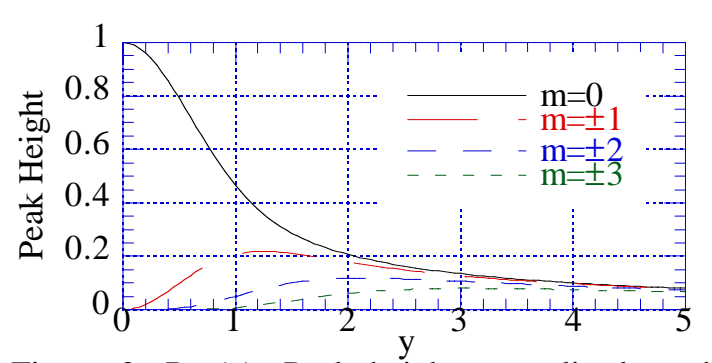

Figure 2: $\mathrm{R}_{\mathrm{m}}(\mathrm{y})$ : Peak height normalized to the peak height of $\mathrm{m}=0$ at $\mathrm{y}=0$.

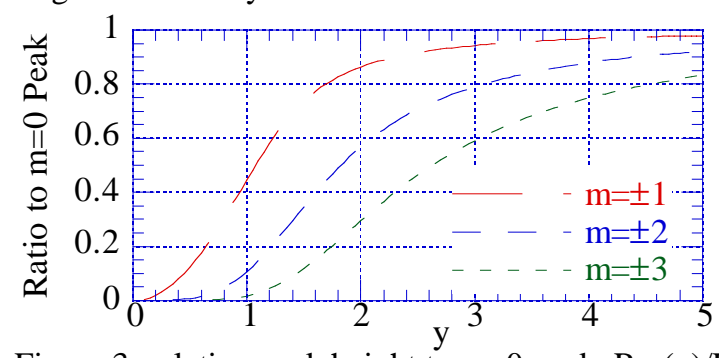

Figure 3: relative peak height to $\mathrm{m}=0$ peak, $\mathrm{R}_{\mathrm{m}}(\mathrm{y}) / \mathrm{R}_{0}(\mathrm{y})$.

\section{ENERGY SPREAD MEASUREMENT}

Using the result above, we measured the energy spread of the SPring- 8 storage ring by comparing the measured frequency response of betatron motion with the result above. The total current is $1 \mathrm{~mA}$ that is low enough to avoid collective effects such as potential distortion by wake field, energy spread increase by so-called microwave instability or single-bunch head-tail effects. These effects distort the betatron motion or synchrotron motion from simple harmonic oscillation that we assume in this discussion.

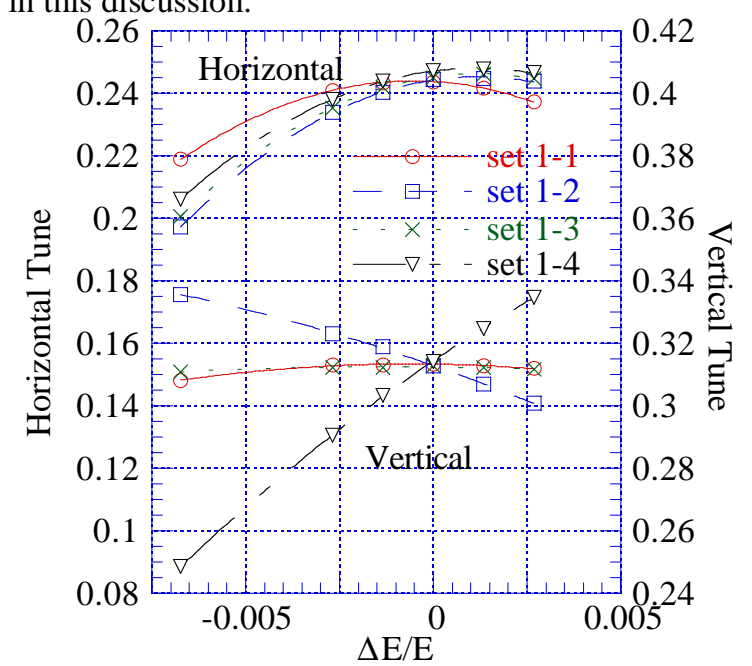

Figure 3: Energy dependence of tune for four settings of sextupole magnets

Fig. 3 shows the dependence of tunes on energy shift with different sextupole setting and shows the points where we observed the frequency response. The results is shown in Fig. 4 and Fig. 5 and we have $1.0 \times 10^{-3}$ for the value of the energy spread of the ring and this value is close to the calculated value by MAD, $1.08 \times 10^{-3}$. The 
momentum compaction factor is required to calculate the energy shift from the RF acceleration frequency shift. The measured value from synchrotron frequency measurement is $1.46 \times 10^{-4}$ and is the same as the design value. The energy damping partition is changed by the energy shift. We measured and calculated by MAD and obtained $\sim 1.8$ at the energy shift $-0.5 \%$. This value increases $6 \%$ of the energy spread.

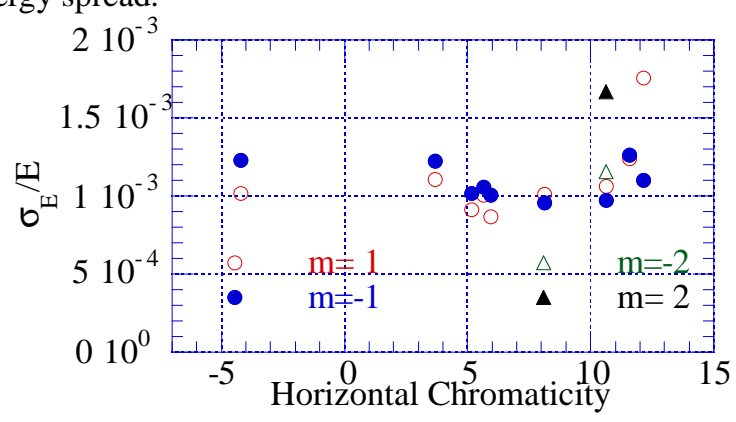

Figure 4: Energy spread obtained from measured data of relative peak height of synchrotron sideband in frequency

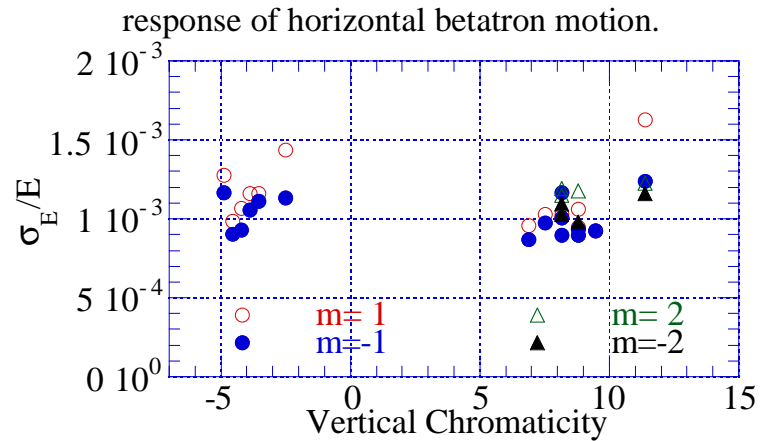

Figure 5: Energy spread obtained from measured data of relative peak height of synchrotron sideband in frequency response of vertical betatron motion.

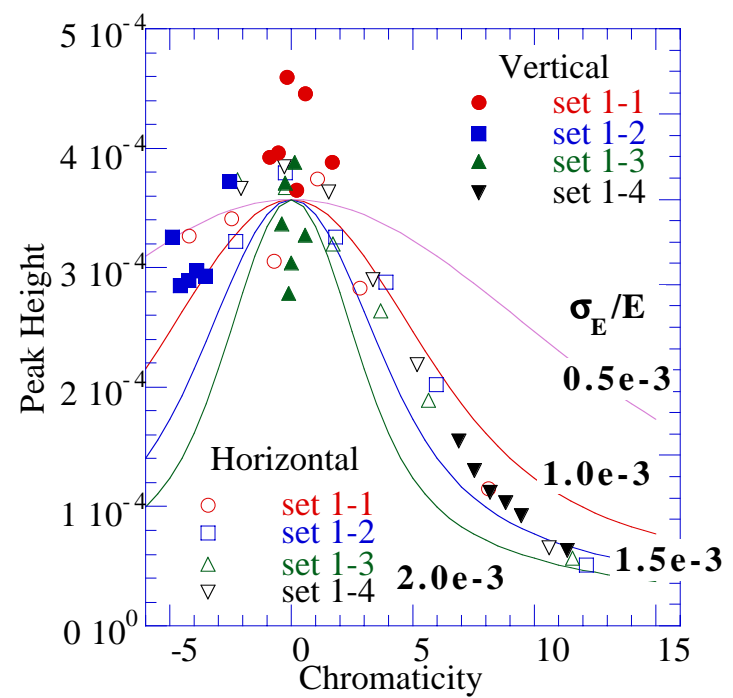

Figure 6: Peak Height of $m=0$ peak(main peak) vs chromaticity. Lines show the calculated value for energy spread shown in Figure.

\section{SUPPRESSION OF INSTABILITY}

The mechanism of reduction of strength of transverse coupled-bunch instabilities is the same as that of the reduction of the height of the main peak of betatron motion. Using the Sacherer's integral equation of frequency domain[1] assuming that the frequency shift is small enough and the coupling of the azimuthal mode is negligible, which is usual case for low bunch current, we have the same expression as Eq.(10) for reduction of the growth rate of the instability using $y=\left(\frac{\omega_{f} \alpha}{\omega_{s}}+\frac{\omega_{0} \xi}{\omega_{s}}\right) \sigma_{\delta}$ where $\omega_{\mathrm{f}}$ is the frequency of the instability resonance such as cavity higher order mode frequency. Calculation for the sample cavity transverse impedance, $R_{t}=30 \mathrm{M} \Omega$, with $\omega_{\mathrm{f}}=6.3 \times 10^{9}, \omega_{\mathrm{s}}=1.095 \times 10^{4}, \omega_{0}=1.31 \times 10^{6}$ and momentum compaction factor $\alpha=1.46 \times 10^{-4}$, is shown in Fig. 7 with the multi-particle simulation[2].

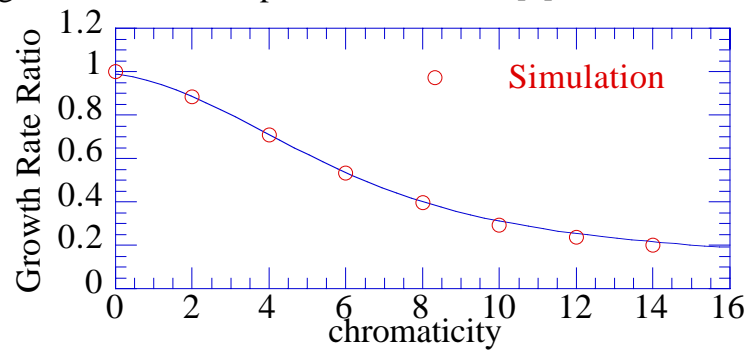

Figure 7. Reduction of growth rate of transverse coupled-bunch instabilities driven by the model impedance.

We did not systematic measurement of the reduction of growth rate. However, we observed following phenomena.

1) When horizontal chromaticity was zero, the threshold current of horizontal coupled-bunch instability driven by higher order resonance mode of acceleration cavities was 50mA. After we increased the chromaticity to 7 , we could store $100 \mathrm{~mA}$ which is required for user operation.

2) When vertical chromaticity was zero, the number of in-vacuum type insertion devices of minimum gap was limited to 4 because of the vertical coupled-bunch instability driven by their resistive-wall impedance. After we increased the chromaticity to 6 , we could increase to more than 8 .

\section{REFERENCES}

[1] Eq. (6.191)-(6.192) in a text book, "Physics of Collective Beam Instabilities in High Energy Accelerators," A.W. Chao, Wiley, 1993, and using $\alpha_{11}$ ' = $\delta_{11}$,

[2] CISR, T. Nakamura, http://acc-web.spring8.or.jp/ nakamura 